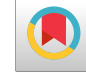

\title{
Early-Onset Meningitis with Group B Streptococcus: The Case of a Female Neonate with Brain Abscess in Iran
}

\author{
Ziba Mosayebi, ${ }^{1}$ Mahboobeh Mohammadi, ${ }^{1,}$ Marzieh Mohammadi, ${ }^{2}$ Ali Jafari, ${ }^{1}$ and Amir Hossein \\ Movahedian $^{3}$ \\ ${ }^{1}$ Department of Pediatrics, Children's Medical Center, Tehran University of Medical Sciences, Tehran, Iran \\ ${ }^{2}$ Department of Immunology, Tehran University of Medical Sciences, Tehran, Iran \\ ${ }^{3}$ Associate Professor of Pediatric Cardiology, Bahrami Children Hospital, Tehran University of Medical Sciences, Tehran, Iran \\ "Corresponding author: Mahboobeh Mohammadi, MD, Gharib St, Children's Medical Center, Tehran, Iran. Tel: +98-2161472781, Fax: +98-2166929235, E-mail: \\ m_m361kord@yahoo.com
}

Received 2017 May 09; Revised 2017 July 25; Accepted 2017 September 20.

\begin{abstract}
Introduction: Group B streptococcus (GBS) is a leading cause of neonatal sepsis and meningitis. Although late-onset GBS meningitis is the most common form of the disease, it may happen earlier, like just a few hours after birth, known as the early-onset. GBS meningitis is associated with $30 \%$ mortality rate among infants. Of those who stay alive, $25 \%$ of them will develop adverse neurological conditions. Herein, we report a case of early-onset GBS meningitis in a female infant with brain abscess.

Case Presentation: A 10-hour old female was admitted to the neonatal intensive care unit (of the University of Tehran) for poor feeding, irritability, lethargy, and seizure. Sepsis work-up and lumbar puncture were performed. The treatment was started with vancomycin, cefotaxime and phenobarbital. The GBS was isolated from both blood and CSF cultures. A cranial sonography revealed intraparenchymal hemorrhage. The infant's hospital course was complicated by recurrent and intractable seizures. The brain MRI of the patient showed micro-abscess. After six weeks of medical therapy, the patient was discharged from the hospital in a good condition. Neurological evaluations of the patient, conducted two and five months after her birth, showed normal results.

Conclusions: Neonates with GBS meningitis are prone to adverse neurological conditions, including brain abscess. Although GBS is a normal flora in the genitourinary tracts of 15 to 30\% of women, routine screening and treatments of the GBS-positive women during their course of pregnancy could reduce such serious complications.
\end{abstract}

Keywords: Brain Abscess, Group B Streptococci, Sepsis, Neonate, Meningitis

\section{Introduction}

Group B Streptococcus (GBS) is an important cause of neonatal sepsis and meningitis, with a high mortality and morbidity rate (1). Neonatal GBS infection can be categorized into two forms: the early-onset and the late-onset. The former occurs within the first week of life, whereas the latter occurs between one week and three months of age (2). Maternal GBS colonization in the vagina-detected among 10 to $30 \%$ of pregnant women-is a major risk factor for neonatal GBS infections (3). Intrapartum antibiotic prophylaxis can reduce the incidence of the early-onset GBS infections but not that of the late-onset. Although the GBS meningitis is most common in late-onset disease, it can happen earlier, e.g. like after the birth. GBS meningitis is associated with $30 \%$ mortality among infants and the survivors are at risk of developing neurological sequelae (4). Although GBS meningitis is a serious manifestation of lateonset disease, the early-onset form of the disease is, equally, important. This paper reports the case of early-onset GBS meningitis in a female infant with brain abscess from Iran.

\section{Case Presentation}

A term female infant with a birth weight of $3 \mathrm{~kg}$ was born through a prolonged and difficult vaginal delivery, with a normal Apgar score. The mother had an uneventful pregnancy and no GBS screening had been performed on her during the course of her pregnancy. Ten hours after her birth, the infant was admitted to NICU (of the University of Tehran) for poor feeding, irritability, and seizure. The treatment started with ampicillin and cefotaxime, along with Phenobarbital. The blood and CSF cultures of the infant were both positive for the GBS in the first round of examination. According to the antibiogram of the patient, the GBS was sensitive to penicillin, cephazolin, cefotaxime and vancomycin. No intermediate and resistant patterns were reported. Due to the poor condition of the patient and 
the availability of antibiogram, antibiotics were changed to vancomycin and cefotaxime. Response to the treatment and poor condition of the patient, confirmed to continue the use of the two above-mentioned drugs. For evaluating the treatment process, the CSF analysis was repeated, which became negative. Yet, because of the deterioration and progression of the disease, complicated by recurrent and intractable seizures, on the one hand, and for excluding the complications of meningitis such as ventriculitis on the other hand, the blood and CSF analyses were repeated with the consultation of a pediatric with expertise on infectious diseases (Table 1). Cranial ultrasound revealed a hypoechoic area with dimensions of $16 \times 17$ in the frontal horn of the lateral ventricle and the right caudate. Because of the clinical deterioration and the recurrence of seizure, a brain CT scan was conducted, which showed low attenuation areas around the right lateral ventricle and at caudate head, which was suggestive of hypoxic ischemic insult (Figure 1). The brain MRI of the patient demonstrated bilateral periventricular cyst formation, mainly on the right side of the caudate head and putamen, which represented brain abscess (Figures 2 and 3 ). The neurosurgical consultant recommended the continuation of the medical treatment. After six weeks of antibiotic therapy, the patient was discharged from the hospital with a good general condition. Neurological evaluation of the patient, conducted two and five months after the birth of the infant showed normal results.

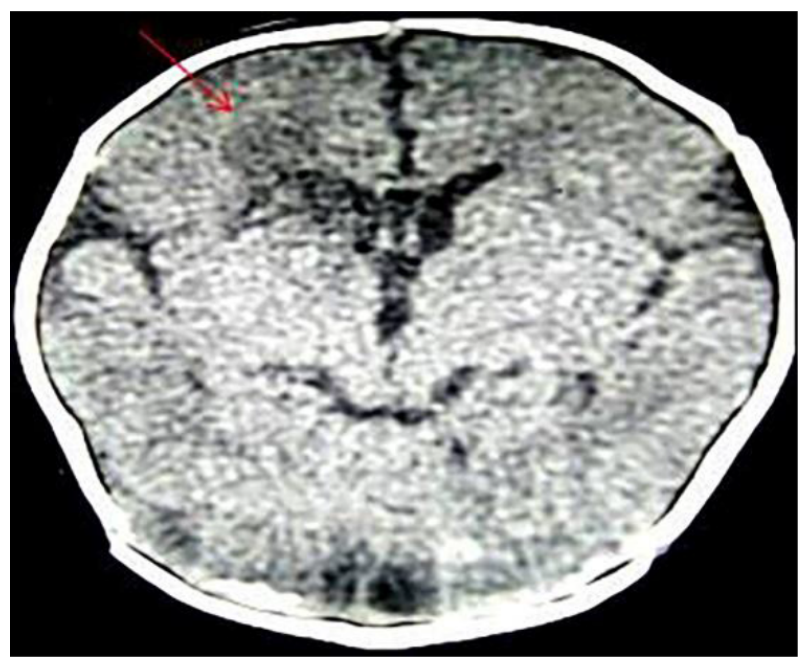

Figure 1. Brain CT Scan: Low Attenuation Areas Around the Right Lateral Ventricle and at Caudate Head Suggestive of Hypoxic Ischemic Insult

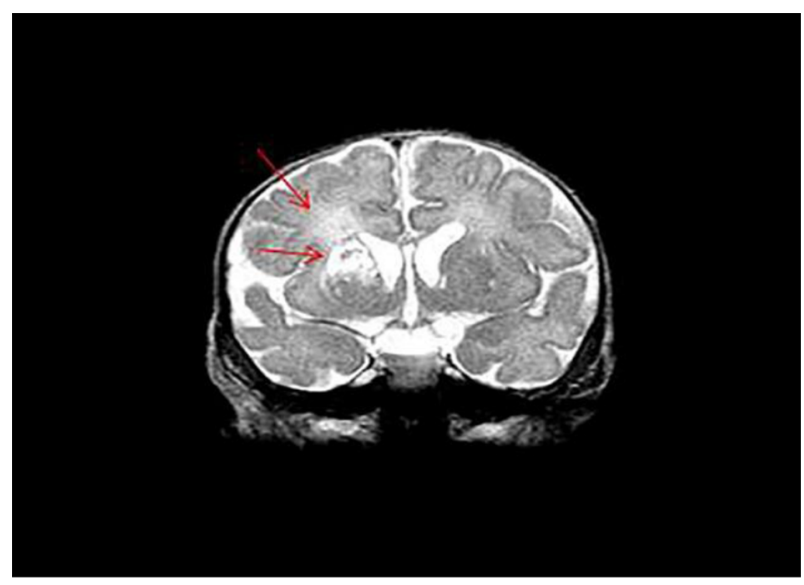

Figure 2. Braim MRI: Bilateral Periventricular Cyst Formation Mainly on the Righ Side of the Caudate and Putamen

\section{Discussion}

Neonates are at a greater risk of meningitis than any other age groups. Although the early-onset meningitis as the consequence of GBS is not common, the presentation of meningitis in the first week of life in the case of our patient suggests maternal vertical transmission.

GBS is a normal flora in the genitourinary tracts of 15 to 30 percent of women. Strategies for prenatal screening and intrapartum antibiotic prophylaxis could decrease the incidence of the early-onset GBS infections by 70 to 80 percent, though the late-onset disease incidence remains unchanged $(5,6)$. In Iran and some other countries, it is not routine to screen GBS colonization in pregnant women during the perinatal care $(6,7)$. Therefore, pregnant women who carry GBS can transfer it to their infants. About half of the infants born from carrier mothers have no symptoms at birth; however, $1 \%$ of them have invasive infections such as sepsis, meningitis, pneumonia, septic arthritis, among others (8). In the case of the present study, the GBS screening was not performed on the mother. The two invasive infections (i.e. sepsis and meningitis) developed in the neonate during the first hours of the neonate's life. Prophylactic measures in the mother might have prevented these serious manifestations in the neonate. Although GBS meningitis is more common as a late-onset infection, it can, sometimes, develop earlier and even just a few hours after birth (8). Coni et al., for example, reports six cases of GBS meningitis, of which three infants had earlyonset infection; GBS screening was performed for all of them except one and the results were negative. There is a high rate of identification of carrier state (about 54\% $84 \%$ ), but some false negative results should be considered 
Table 1. Blood and CSF Analysis

\begin{tabular}{|c|c|c|c|c|}
\hline \multicolumn{2}{|l|}{ Variables } & \multicolumn{3}{|c|}{ Days from Admission } \\
\hline \multicolumn{5}{|l|}{ CBC } \\
\hline & First & Fourth & Tenth & Seventeenth \\
\hline WBC & 16400 & 13700 & 15900 & 13400 \\
\hline PMN, \% & 68 & 43 & 42 & 29 \\
\hline PLT & 202000 & 231000 & 402000 & 492000 \\
\hline CRP & 192 & 67 & 49 & 3 \\
\hline \multicolumn{5}{|l|}{ CSF } \\
\hline & First & Fourth & Eleventh & Twenty-second \\
\hline WBC & 7500 & 3 & 19 & 3 \\
\hline PMN, \% & 80 & 2 & 5 & 0 \\
\hline $\operatorname{Pr}$ & 152 & 180 & 60 & 63 \\
\hline Glucose & 18 & 67 & 29 & 40 \\
\hline
\end{tabular}

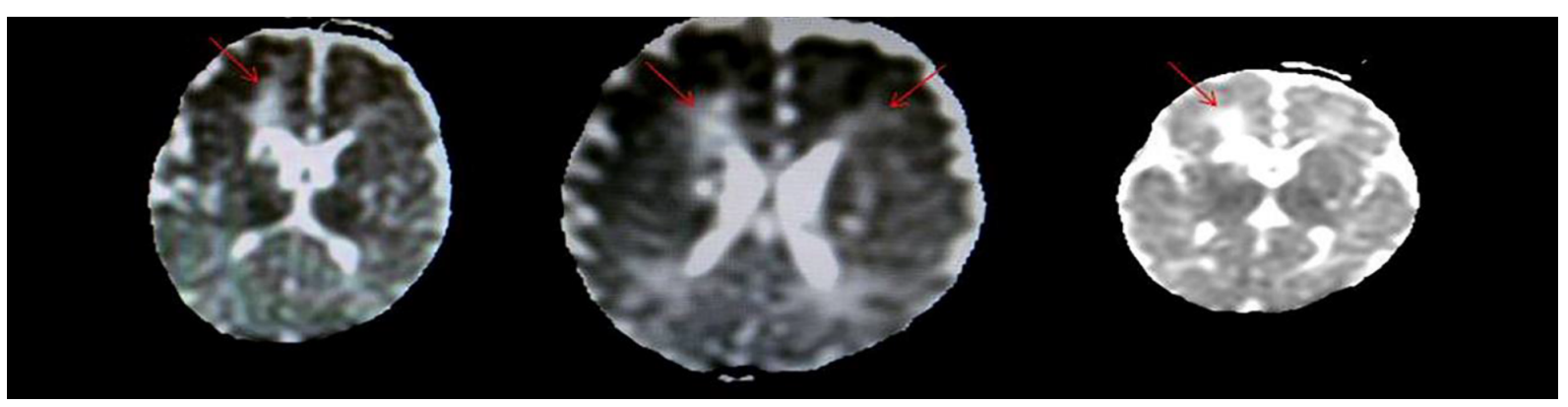

Figure 3. Brain MRI: Bilateral Periventricular Cyst and Abscess

(9). Pregnant women with unknown GBS status should be treated with antibiotics at the time of delivery (10).

Due to the serious neurological impacts of GBS meningitis, such as cerebral edema, ventriculitis, cerebritis, subdural effusion, empyema, infarction, hemorrhage and brain abscess, neuroimaging is recommended to detect these complications. Brain abscess is a rare complication of neonatal meningitis. Ultrasound examination is an important tool for the initial diagnosis, although it may show normal results at the initial stages, as seen in the case of our patient. CT scan and MRI are other modalities that may be used to confirm the diagnosis.

A multidisciplinary team-including neurosurgeons and infectious disease specialists-should be involved in the treatment of brain abscess in neonates. Brain abscess can be treated both medically and surgically. The nature, size and anatomical locations of the abscess and the neurological status of the patient can influence the treatment strategy (5). Medical therapy could save the patient from the stress and complications of surgery. Our patient was successfully treated with six weeks of appropriate antibiotic therapy.

Long-term outcomes for children who survive the GBS meningitis reveal that $56 \%$ have normal function. The remaining undergo mild to moderate (25\%) or to severe (19\%) neurodevelopmental impairments (4). Our patient was discharged in a good condition. Neurological evaluations of our patient at two and five months of birth demonstrated normal findings, but long-term follow-up is needed.

\subsection{Conclusion}

Neonates with GBS meningitis are prone to adverse neurological outcomes, including brain abscess. Although GBS is a normal flora in the genitourinary tracts of 15 to30 percent of women, routine screenings and treatments of the GBS-positive women could reduce such serious complications. 


\section{References}

1. Kumasaka S, Shima Y, Mine M, Nakajima M, Migita M. Neonatal case of late-onset sepsis involving group B Streptococcus type Ib. J Nippon Med Sch. 2013;80(5):384-6. doi: 10.1272/jnms.80.384. [PubMed: 24189357].

2. Faro S, Brehm B, Smith F, Mouzoon M, Greisinger A, Wehmanen O, et al. Screening for group B streptococcus: a private hospital's experience. Infect Dis Obstet Gynecol. 2010;2010. doi: 10.1155/2010/451096. [PubMed: 20689734].

3. Ko TJ, Hsieh WS, Hsueh PR, Chou HC, Lu CY. Late-onset group B streptococcal meningitis in a neonate with early antibiotic prophylaxis. Pediatr Neonatol.2010;51(4):242-4. doi:10.1016/S1875-9572(10)60046-0. [PubMed: 20713290].

4. Libster R, Edwards KM, Levent F, Edwards MS, Rench MA, Castagnini LA, et al. Long-term outcomes of group B streptococcal meningitis. Pediatrics. 2012;130(1):e8-15. doi: 10.1542/peds.2011-3453. [PubMed: 22689869].

5. $\mathrm{Lu} \mathrm{CH}$, Chang WN, Lui CC. Strategies for the management of bacterial brain abscess. J Clin Neurosci. 2006;13(10):979-85. doi: 10.1016/j.jocn.2006.01.048. [PubMed: 17056261]

6. Eskandarian N, Neela V, Ismail Z, Puzi SM, Hamat RA, Desa MN, et al. Group B streptococcal bacteremia in a major teaching hospital in Malaysia: a case series of eighteen patients. Int J Infect Dis. 2013;17(9):e777-80. doi: 10.1016/j.ijid.2013.01.011. [PubMed: 23453715].

7. Shirazi M, Abbariki E, Hafizi A, Shahbazi F, Bandari M, Dastgerdy E. The prevalence of group B streptococcus colonization in Iranian pregnant women and its subsequent outcome. Int J Fertil Steril. 2014;7(4):267-70. [PubMed: 24520495].

8. Coni E, Marcialis MA, Pintus MC, Irmesi R, Masile V, Fanos V. Group B Streptococcal Meningitis: A Description of Six Case Reports. Int J Clin Pediatr. 2015;4(1):127-36. doi: 10.14740/ijcp179w.

9. Natarajan G, Johnson YR, Zhang F, Chen KM, Worsham MJ. Real-time polymerase chain reaction for the rapid detection of group B streptococcal colonization in neonates. Pediatrics. 2006;118(1):14-22. doi: 10.1542/peds.2005-1594. [PubMed:16818544].

10. Vaciloto E, Richtmann R, Costa HP, Kusano EJU, Almeida M, Amaro ER A survey of the incidence of neonatal sepsis by group B Streptococcus during a decade in a Brazilian maternity hospital. Braz J Infect Dis. 2002;6(2):55-62. doi: 10.1590/s1413-86702002000200001. 